\title{
IMMUNOLOGIC ASPECTS OF PEDIATRIC TONSIL AND ADENOID DISEASE
}

\author{
A. Zamfir-Chiru-Anton ${ }^{1}$, D.C. Gheorghe ${ }^{2}$ \\ 1 "Grigore Alexandrescu" Emergency Clinical Hospital for Children, Bucharest \\ 2"M.S. Curie" Emergency Clinical Hospital for Children, \\ "Carol Davila" University of Medicine and Pharmacy, Bucharest
}

\begin{abstract}
The mechanisms responsible for adenoids and tonsils hypertrophy still remain a research debate, but lots of published papers show that the proliferative processes in obstructing pharyngeal tonsils are differently regulated versus those in recurrent infectious disease.

Studying the immunology of these lymphoid organs allows a better understanding and, eventually, the finding of specific novel targets for the treatment of lymphoid hypertrophy and prevention of complications usually associated with it: cardiovascular, neurological and behavioral.
\end{abstract}

Keywords: pediatric tonsillitis, immune responses

Obstructive sleep apnea is a frequent syndrome that is found in 1 to $3 \%$ of pediatric respiratory diseases (1). It is a disease with recurrent bouts of respiratory arrest during sleep, followed by pulmonary hypoventilation, hypoxemia and hypercapnia (2). The main responsible causes in children are obesity and tonsils and/or adenoids chronic enlargement (3). The palatine tonsils are an immunologic defense system, contributing to immune antibody response and immune cells proliferation. They can also become chronically infected and become a source for recurrent local acute disease.

The severity and the prognosis of obstructive sleep apnea can be demonstrated by polysomnography. Friedman proposed a clinical staging, considering the anatomic elements that can be assessed on physical examination. It can eventually predict the sleep obstructing degree for a certain patient (4).

- Stage I - the uvula and the tonsils are both clearly visible at inspection (type I) or just the uvula is visible (type II) combined with type III (tonsils larger than the medial edge of posterior pillars) or IV (tonsils touching each in the midline) tonsils hypertrophy;

- Stage II - uvula and tonsils invisible at simple clinical exam (type III) or only hard palate visible (type IV) combined with type III or IV tonsils hypertrophy;
- Stage III - tonsils hypertrophy less obvious; they are completely between the pillars (type I) or discretely enlarged (type II).

All patients having a body index mass of over 40 are belonging to stage III.

Some published studies have focused on lab cultures of cell lines harvested from hypertrophied tonsils or adenoids; the lab conditions were close to the in vivo situation (5). They showed the role of inflammation as a trigger for adenoids and tonsils enlargement encountered in sleep apnea patients and also in recurrent infectious tonsillitis. It seems that the immune response in patients having large obstructive lymphoid tissues is different from that seen in normal children. The leukotriene receptor concentration (CysLTR1 and CysLTR2) is higher in hypertrophied adenoids than in patients with recurrent infectious disease (6). The receptors are actively synthesized in all lymphoid tissues explaining the relative efficiency of leukotriene receptor antagonists (like Montelukast) efficiency in diminishing tonsils volume, with significant aleviation of sleep apnea as well (7).

Some research has been performed on steroid receptors in tonsilar samples extracted from both hypertrophy and recurrent infections patients (8). Higher receptor concentrations were found in large 
obstructive tonsils as opposed to recurrent disease ones. That could also give an explanation of local (nasal) steroid efficiency for controlling sleep apnea symptoms (9).

Other research focused on molecular basis involved in tonsilar response to antigens and elicited the role of macrophage migration inhibitory factor (MIF) in the inflammatory process. It is a protease associated with hypophyseal-hypothalamic-adrenal axis and could be a potential target using medication aimed to lower the antigen specific responses in tonsilar or adenoid tissues (10). It also inhibits glucocorticoid effects. Some authors reported high concentrations of MIF in patients with obstructive sleep apnea and associated the fact with the clinical severity of the disease (11).

The allergic reactions can contribute to genesis of enlarged tonsils and adenoids. Some researchers included food allergy patients in their studies, looking for a possible association of proteins like casein, alpha-lactalbumin, beta-lactoglobulin $(12,13)$ or ovalbumin with allergic respiratory responses. The allergen epitopes are specifically bound by immunoglobulins of types IgE, IgG4 and IgA. The consequent inflammatory reaction after $\operatorname{IgE}$ specific binding is correlated with allergen type. Tolerance to a specific antigen can occur and contribute to $\mathrm{IgE}$ altered/lowered inflammatory reaction in time. On the opposite, IgG4 maintain their epitope binding specificity indefinitely. They can confer allergic tolerance to some antigens due to their competitive binding to the same peptidic fractions as $\operatorname{IgE}(14)$. These facts could be of use as prognostic markers or for developing new treatment protocols, by means of obtaining higher specific IgG4 protective levels in certain patients (15).

Some authors measured IFN $\alpha$, IFN $\gamma, \operatorname{IgA}$, IgG, $\mathrm{IgE}$ in patients saliva and also researched lymphocites subpopulations in biopsy samples from EBV, HPV, CMV tonsilitis that were diagnosed and confirmed by PCR testing (16). Complicated forms of tonsilitis, with toxic and allergic reactions were recorded to have less IgG and higher IgE concentrations. In that view, one can propose a substitution therapy with antibodies (intravenous immunoglobulins). Correction of an abnormal immune response would also be possible by administering IFN $\alpha$, IFN $\gamma$ and bacterial extracts. It has already been published evidence about a direct correlation between IFN-gamma levels and Th2 cells involved in inflammatory reactions (17).

Molecular studies of atopic dermatitis focused on the enzymes involved in epithelial breaking (18). There have been described proteinase activat- ed receptors (PAR 1 and 2) that play critical roles in the pro/anti inflammatory responses of the respiratory mucosa as well (19). Their activation mediate IL1 and IL8 secretion, with subsequent increased vascular permeability and leukocyte local response. T-antigen presenting cells initiate the inflammatory reaction and activate Th2 cells. That is quickly followed by interferon (IFN)-gamma and IL3, IL4, IL9 and IL13 secretion, with mast cells, eosinophils and basophils activation. Epidermal dendritic cells produce IL1, IL6 and TNF-alpha. The evolution of inflammation can pass to a chronic stage if IL12 and IL18 are involved, leading to a Th1 mediated immune response (20).

The Th1 mediated immune response is intense and is at first generated by high concentrations of IFN-gamma and TNF-alpha. An immune antibody reaction follows (Th2 mediated), less obvious and as a consequence of IL6 and IL4 accumulation. IL6 is high in tonsillar tissues but IL4 predominates in the blood (21).

Other detailed aspects of the inflammatory process in allergic rhinitis have been elicited by some researchers. Th1 and IFN-gamma can suppress Th2 production (22). The same action is attributable to IL18 (23). Levamisole, a current antihelmitic drug, can alleviate allergic rhinitis in mice by raising the IL18 levels (24).

TNF-alpha is a proinflammatory cytokine and also a chemokine for granulocytes. Its production can be stimulated by administering ovalbumin-recombinant peptides (or similar allergens). Its role is demonstrated by monoclonal antibodies (Infliximab) therapy which inhibits TNF-alpha secretion and decreases the allergic responses, Th2 mediated (25).

SHP-1 is a protein regulator of Th2 pathway (17). It is an important modulator in upper and lower airways. It binds to IL4 receptors and inactivate them, thus altering the immune response Th2 mediated. Considering other IL4 functions (stimulation of eosinophils, mast cells and Th2 cells differentiation, enhancing macrophages ability to present antigen, B-cells differentiation) we can assume the importance of SHP-1 protein in the inflammatory reaction.

From a histologic point of view, hypertrofied tonsils have a thin epithelium that is in close contact with the lymphoid tissue beneath it. Lymphoid follicles are large, hyperplastic, with scarse interfollicular tissue. In recurrent tonsilitis, the covering epithelium has metaplasia, with some thick keratin structure. The lymphoid follicles are smaller, with clear margins. Fibrosis is abundant around the follicles and the crypts contain cellular debris. 
In enlarged obstructive tonsils there have been demonstrated multiple types of immune cells inside the large lymphoid follicles. T-cells predominate along with lower numbers of B-cells. The B-cell response is more obvious instead in patients with recurrent tonsillitis.

The use of immuno-fluorescence on tonsillar samples confirmed the presence of B-cells in follicular regions with T-cell predominance in the extrafollicular regions of obstructive/enlarged organs. The B and T-cell (cysteinyl LT) receptor distribution in these patients showed variate concentrations. Some can discuss the possibility of those receptors as targets in cases of obstructive hypertrophied tonsils (7).

Mast cells are distributed in the interfolicullar and perivascular regions. They are more dense in tonsillar tissue from allergic patients than in normal subjects. Their activation seem to be mediated by CD4+ (Th2) cells (26).

Other research focused on citokines concentration in peripheral blood. No differences were noted in regard to the type of chronic inflammation of the tonsil. Still, the tonsil IFN $\gamma$ concentration is 3-5 times higher in recurrent infectious disease compared to just enlarged lymphoid tissue. That fact suggests a immune system activation deficiency: higher blood concentration of IL6, IFN $\gamma$, TNF $\alpha$ and lower concentration of IL10 (antiinflammatory). In both types of tonsilitis, Th1-mediated citokine production (TNF $\alpha$ and IFN $\gamma$ ) is higher than Th2-mediated production (IL6 şi IL4), explaining a predominant cell response as opposed to the antibody production response (21). Recently, a T-helper cell subgroup has been described (Th17), producing proinflammatory cytokines (IL17) and differentiated distinctly from Th1 and Th2. They seem implicated in antibacterial immunity (27).

Group A streptococcus (GAS) has long been advocated as the main cause for recurrent tonsillitis. Some studies researched the immunization process against this bacteria, using ovalbumin GAS (bacteria presenting the proteic epitope on its surface) (26). CD4+ (T cells) mediated the immune reaction. Their activation was located within nasal mucosa or systemic lymph nodes and spleen, depending on the route used to administer the antigen. When nasal antigen presentation was used, high levels of CD4+, IL17 and IFN-gamma could be elicited, locally. Human immune system does not develop long time protection against GAS after a first/single streptococcal infection. The immune re- action is a short one, Th17 mediated (28). To efficiently induce immunity for mucosal GAS, longer and repeated Th17 production by $\mathrm{CD} 4+$ cells differentiation is needed (29). The practical implications of this evidence remain to be discovered.

The streptococcal affinity for tonsillar tissue has been reported to be a consequence of local TGF $\beta$ and IL6 higher levels, in some patients.

Specific immune therapy should address 2 pathways:

- T-antigen presenting cells blocking;

- IgG4 expression stimulation and mast cells, eosinophils and $\operatorname{IgE}$ inhibiting.

The mechanisms used to achieve these goals and obtain desensitisation are augmenting IL10 and TGF-beta levels. Also, T-cell specific immunoglobulin therapy should suppress $\mathrm{Th} 2$ activity and IgE accordingly and enhance IgG4 production (30).

Some studies addressed the issue of IL6 and IL8 concentrations, in both types of tonsil disease. Differences were recorded as well: IL8 is high in recurrent disease and IL6 (along with IFN-alpha) is high in enlarged, obstructive tonsils (5). Also, genetic studies demonstrated an association between some IL6 gene polymorphysm $(-174 \mathrm{G} / \mathrm{C})$ and the presence of obstructive tonsils (31).

The tonsils with recurrent infections show a high degree of superficial fibrosis and a high concentration of memory lymphocites (CD45R0+), Bcells (CD20+), T-cytotoxic cells (CD8+) (32). The IgG, IgA, IgM and TNF- $\alpha$ concentrations along the tonsilar crypts are also high, possibly because of an inflammatory reaction maintained by local chronic persistence of bacterial antigens (32). Immune response Th1 mediated can be influenced by differences of local concentration of TNF $\alpha$ and IL4. Distant inflammatory reactions throughout the body can be explained by an abnormal immune response with T-cells (CD8+) superproduction along with low expression of PD-1 (programmed cell death-1) and FOXp3 (fork head box protein-3) (33).

\section{CONCLUSIONS}

A better knowledge of the immune responses, according to their triggers, allows us developing new treatment options and addressing both installed disease and its prevention. Although far from perfect understanding, the complex molecular mechanisms involved in tonsilar enlargement and chronic tonsilar infection allow a more efficient way of solving a very old pediatric disease. 


\section{REFERENCES}

1. Lumeng J.C., Chervin R.D. Epidemiology of pediatric obstructive sleep apnea. Proc Am Thorac Soc, 2008, 5(2): p. 242-52.

2. Dayyat E., Kheirandish-Gozal L., Gozal D. Childhood Obstructive Sleep Apnea: One or Two Distinct Disease Entities? Sleep Med Clin, 2007, 2(3): p. 433-444.

3. Kang K.T., et al. Body weight status and obstructive sleep apnea in children. Int J Obes (Lond), 2012, 36(7): p. 920-4.

4. Friedman M., Ibrahim H., Bass L. Clinical Staging for SleepDisordered Breathing. Otolaryngology - Head and Neck Surgery, 2002, 127(1): p. 13-21.

5. Serpero L.D., et al. A mixed cell culture model for assessment of proliferation in tonsillar tissues from children with obstructive sleep apnea or recurrent tonsillitis. Laryngoscope, 2009, 119(5): p. 1005-10.

6. Zhu M.H., et al. (Expression of CysLTR-1 and CysLTR-2 in adenoid tissues from children with adenoid hypertrophy). Zhongguo Dang Dai Er Ke Za Zhi, 2015, 17(2): p. 159-63.

7. Tsaoussoglou M., et al. Expression of leukotriene biosynthetic enzymes in tonsillar tissue of children with obstructive sleep apnea: a prospective nonrandomized study. JAMA Otolaryngol Head Neck Surg, 2014, 140(10): p. 944-50.

8. Malakasioti G., et al. Low morning serum cortisol levels in children with tonsillar hypertrophy and moderate-to-severe OSA. Sleep, 2013, 36(9): p. 1349-54.

9. Edwards K.M., et al. Obstructive sleep apnea and neurocognitive performance: the role of cortisol. Sleep Med, 2014, 15(1): p. 27-32

10. Mizue Y., et al. Role for macrophage migration inhibitory factor in asthma. Proc Natl Acad Sci USA, 2005, 102(40): p. 14410-5.

11. Edwards K.M., et al. Macrophage migratory inhibitory factor (MIF) may be a key factor in inflammation in obstructive sleep apnea. Sleep, 2011, 34(2): p. 161-3.

12. Wal J.M. Bovine milk allergenicity. (1081-1206 (Print)).

13. Jarvinen K.M., et al. B-cell epitopes as a screening instrument for persistent cow's milk allergy. (0091-6749 (Print)).

14. Wachholz P.A., et al. Inhibition of allergen-lgE binding to $B$ cells by IgG antibodies after grass pollen immunotherapy. J Allergy Clin Immunol, 2003, 112(5): p. 915-22.

15. Francis J.N., et al. Grass pollen immunotherapy: IL-10 induction and suppression of late responses precedes IgG4 inhibitory antibody activity. J Allergy Clin Immunol, 2008, 121(5): p. 1120-1125 e2.

16. Miramontes H.P., et al. Prevalence of microorganisms and immunoglobulins in children with tonsillar hypertrophy and adenoiditis. Int Arch Otorhinolaryngol, 2014, 18(3): p. 311-5.

17. Cho S.H., et al. Regulation of Nasal Airway Homeostasis and Inflammation in Mice by SHP-1 and Th2/Th1 Signaling Pathways. PLOS ONE, 2014, 9(8): p. e103685.
18. Cork M.J., et al. New perspectives on epidermal barrier dysfunction in atopic dermatitis: gene-environment interactions. J Allergy Clin Immunol, 2006, 118(1): p. 3-21; quiz 22-3.

19. Kawabata A., Kawao N. Physiology and pathophysiology of proteinase-activated receptors (PARs): PARs in the respiratory system: cellular signaling and physiological/pathological roles. J Pharmacol Sci, 2005, 97(1): p. 20-4.

20. Bieber T. Atopic dermatitis. N Engl J Med, 2008. 358(14): p. 1483-94.

21. Aznabaeva L.F., N.A. Aref'eva, (Immunological aspects of chronic tonsillitis). Vestn Otorinolaringol, 2013(4): p. 4-9.

22. Cohn L., et al. T helper 1 cells and interferon gamma regulate allergic airway inflammation and mucus production. J Exp Med, 1999, 190(9): p. 1309-18.

23. Savolainen J., et al. Increased expression of allergen-induced in vitro interleukin-10 and interleukin-18 mRNA in peripheral blood mononuclear cells of allergic rhinitis patients after specific immunotherapy. Clin Exp Allergy, 2004, 34(3): p. 413-9.

24. Wang H., et al. Topical levamisole hydrochloride therapy attenuates experimental murine allergic rhinitis. Eur J Pharmacol, 2007, 577(1-3): p. 162-9.

25. Mo J.H., et al. Anti-tumor necrosis factor-alpha treatment reduces allergic responses in an allergic rhinitis mouse model. Allergy, 2011. 66(2): p. 279-86

26. Yokoi H., et al. Comparison of human tonsillar mast cell localization and ultrastructural observations between IgE-mediated allergic and nonallergic donors. Allergy Asthma Proc, 2006, 27(5): p. 415-21.

27. Abbas A.K., Murphy K.M. , Sher A. Functional diversity of helper T lymphocytes. Nature, 1996, 383(6603): p. 787-93.

28. Wang B., et al. Induction of TGF-beta1 and TGF-beta1-dependent predominant Th17 differentiation by group A streptococcal infection. Proc Natl Acad Sci U S A, 2010, 107(13): p. 5937-42.

29. Fan X., et al. Sortase A Induces Th17-Mediated and AntibodyIndependent Immunity to Heterologous Serotypes of Group A Streptococci. PLoS ONE, 2014, 9(9): p. e107638.

30. Lee J., Park C.O., Lee K.H. Specific immunotherapy in atopic dermatitis. Allergy Asthma Immunol Res, 2015, 7(3): p. 221-9.

31. Zhong A., et al. Roles of interleukin (IL)-6 gene polymorphisms, serum IL-6 levels, and treatment in obstructive sleep apnea: a meta-analysis. Sleep Breath, 2015.

32. Semberova J., et al. The immune status in situ of recurrent tonsillitis and idiopathic tonsillar hypertrophy. Bratisl Lek Listy, 2013, 114(3): p. 140-4.

33. Meng H.X., et al. Immunoglobulin and CD8(+) T-cell distribution in histologically distinctive tonsils of individuals with tonsillar focal infection. Acta Otolaryngol, 2015, 135(3): p. 264-70. 